\title{
Impact of neutron irradiation on thermal helium desorption from iron*
}

\author{
Xunxiang Hu ${ }^{1}$, Kevin G. Field ${ }^{1}$, Stephen Taller ${ }^{2}$, Yutai Katoh ${ }^{1}$, Brian D. Wirth ${ }^{1,3}$ \\ ${ }^{1}$ Oak Ridge National Laboratory, Oak Ridge, TN 37831 USA \\ ${ }^{2}$ University of Michigan, Ann Arbor, MI 48109 USA \\ ${ }^{3}$ University of Tennessee, Knoxville, TN 37996 USA
}

\begin{abstract}
:
The synergistic effect of neutron irradiation and transmutant helium production is an important concern for the application of iron-based alloys as structural materials in fission and fusion reactors. In this study, we investigated the impact of neutron irradiation on thermal helium desorption behavior in high purity iron. Single crystalline and polycrystalline iron samples were neutron irradiated in HFIR to $5 \mathrm{dpa}$ at $300^{\circ} \mathrm{C}$ and in BOR-60 to $16.6 \mathrm{dpa}$ at $386^{\circ} \mathrm{C}$, respectively. Following neutron irradiation, $10 \mathrm{keV} \mathrm{He}$ ion implantation was performed at room temperature on both samples to a fluence of $7 \times 10^{18}$ $\mathrm{He} / \mathrm{m}^{2}$. Thermal desorption spectrometry (TDS) was conducted to assess the helium diffusion and clustering kinetics by analyzing the desorption spectra. The comparison of He desorption spectra between unirradiated and neutron irradiated samples showed that the major He desorption peaks shift to higher temperatures for the neutron-irradiated iron samples, implying that strong trapping sites for He were produced during neutron irradiation, which appeared to be nm-sized cavities through TEM examination. The underlying mechanisms controlling the helium trapping and desorption behavior were deduced by assessing changes in the microstructure, as characterized by TEM, of the neutron irradiated samples before and after TDS measurements.
\end{abstract}

\section{Introduction}

Iron-based alloys are an important structural material for nuclear energy systems. In the current commercial light water reactors, the major pressure boundary components, such as reactor pressure vessel, pressurizer, steam generator, steam lines, turbine and condenser, are made of either low carbon or low alloy steel. Austenitic stainless steels dominate the core structural materials, as well as serving as the cladding on the inside surface of the reactor pressure vessel and pressurizer [1]. Advanced FeCrAl alloys have been proposed as an important cladding material for the accident tolerant fuel development [2]. Reduced activation ferritic/martensitic (RAFM) steel has been selected as the leading candidate structural material for most ITER test blanket modules and has been under development as the reference structural material for future fusion power reactors for nearly three decades [3]. The extremely hostile service environment of both fission and fusion reactors imposes significant challenges to these iron-based alloys by altering the microstructures and the resultant thermal-mechanical properties. One of the

\footnotetext{
* This manuscript has been authored by UT-Battelle, LLC under Contract No. DE-AC05-00OR22725 with the U.S. Department of Energy. The United States Government retains and the publisher, by accepting the article for publication, acknowledges that the United States Government retains a non-exclusive, paid-up, irrevocable, worldwide license to publish or reproduce the published form of this manuscript, or allow others to do so, for United States Government purposes. The Department of Energy will provide public access to these results of federally sponsored research in accordance with the DOE Public Access Plan (http://energy.gov/downloads/doe-public-access-plan).
} 
primary concerns is the effect of helium $(\mathrm{He})$ in materials, and how the helium interacts with radiation damaged microstructures. In a typical fusion environment, the $14-\mathrm{MeV}$ peaked neutron spectrum will induce on the order of 10 atomic parts per million (appm) of He through $(n, \alpha)$ transmutation reactions per displacement per atom $(\sim 10$ appm $\mathrm{He} / \mathrm{dpa}$ ). The deuterium (D) - tritium (T) fusion reaction also results in the production of a large amount of $3.5 \mathrm{MeV} \mathrm{He}$, which can be implanted into the plasma facing components. In fission reactors, about $90 \%$ of the light nuclei that arise from ternary fission are $\mathrm{He}$, which is also a major decay product of the transmuted actinides. Helium has extremely low solubility in metals and correspondingly, has a strong tendency to interact with microstructural features such as vacancy clusters, voids [4] [5], and dislocation loops [6,7] [8] [9] present in the materials. The concurrent presence of He and radiation induced damaged microstructure promotes He bubble nucleation and growth, and can result in the severe degradation of the structural materials (e.g., swelling, fast creep rupture, embrittlement, etc.) [10].

This inherently multi-scale problem has been intensively studied both theoretically and experimentally for several decades [11] [12]. The kinetics, energetics and thermal stability of He-vacancy clusters in iron [4] [5] [13] [14], and helium interactions with grain boundaries and dislocations [6, 15] [16] [17] [18] in iron have been investigated by using atomistic simulations involving molecular statics/dynamics and ab initio approaches. Kinetic Monte-Carlo [13] and rate theory modeling [19] [20] have been developed to understand the diffusion and growth mechanism of the clusters, therefore, providing data directly compared to experimental results. Dislocation dynamics was used to study issues of hardening induced by He bubble-dislocation interactions [21]. Within the experimental studies, transmission electron microscopy (TEM) was widely used to characterize the radiation damage (i.e., dislocation loops, and cavities, etc.) and $\mathrm{He}$ bubble distribution in Fe and Fe-based alloys [8, 9, 22] [23] [24]. Nuclear reaction depth profiling and secondary ion mass spectroscopy [25] were used to acquire the depth profiling of He and the corresponding diffusion characteristics. Thermal helium desorption spectrometry (THDS) [19, 26, 27] has also been widely used to investigate the He desorption behavior and the corresponding interactions of $\mathrm{He}$ and microstructural features in $\mathrm{Fe}$ and Fe-based alloys followed by coordinated modeling. Positron annihilation spectroscopy [28] has been used to characterize the vacancy defects, which are believed to be strong trapping sites for He, as well as the evolution of He bubbles under various thermal treatments.

However, the overall understanding of He behavior in Fe is still incomplete. Significant open questions remain with respect to the energetics and kinetics of He-defect interactions, systematic characterization of He bubble nucleation and growth, and the consequent mechanical property degradation. One of the primary concerns is the impact of irradiation-induced damage on the He behavior in $\mathrm{Fe}$ and Fe-based alloys. Boron or nickel-containing steels exposed to neutron irradiation have been used to investigate the microstructural evolution and mechanical property changes of materials under simultaneous irradiation damage and He production [29] [30] [31]. Ion irradiation as a surrogate for neutron irradiation and simultaneous He injection have been used to study the He effects in pure Fe or steels [8] $[32,33]$. The most studied case is the He 
implantation on Fe or steels to investigate the near-surface microstructure evolution and the He-defect interactions [26] [34] [27]. The iron samples used in most of the previous studies $[8,35,36]$ were polycrystalline. For the purpose of validating fundamental models of the energetics and kinetics parameters of He-defect interactions in iron, the use of single crystalline iron is more appropriate due to the absence of the grain boundaries, which are strong defect sinks impacting the microstructure evolution [27] [28].

In the present work, we focus on the impact of pre-existing damage microstructure induced by neutron irradiation on the He desorption behavior from high purity single crystalline and polycrystalline Fe samples. Similarities and distinctions between the He desorption spectra of the un-irradiated and neutron-irradiated samples are presented. TEM observations of the neutron irradiated Fe samples before and after TDS measurements provide detailed microstructural information, in order to aid the interpretation of the measured He desorption spectra.

\section{Experiments}

In the present work, both high purity single crystalline and polycrystalline Fe samples were used. The single crystalline Fe samples were purchased from Goodfellow with a dimension of $4.5 \mathrm{~mm}$ in diameter and $1 \mathrm{~mm}$ in thickness, a stated purity of $99.98 \%$, and random orientation. The polycrystalline Fe samples were obtained from ACI Alloys with $3 \mathrm{~mm}$ in diameter and $0.5 \mathrm{~mm}$ in thickness, and a stated purity of $99.98 \%$. Samples were mechanically polished to $1 \mu \mathrm{m}$ grade surface finishing prior to neutron irradiations.

The single crystalline Fe specimen, marked as A4, was exposed to neutron irradiation in the High Flux Isotopes Reactor (HFIR) at Oak Ridge National Laboratory (ORNL). This sample was irradiated at $300^{\circ} \mathrm{C}$ to $5 \mathrm{dpa}$ in the flux trap facility under a fast neutron flux of $1 \times 10^{19} \mathrm{n} / \mathrm{m}^{2} / \mathrm{s}(\mathrm{E}>0.1 \mathrm{MeV})$ corresponding to an appropriate damage rate of $8.1 \times 10^{-7}$ $\mathrm{dpa} / \mathrm{s}$. Neutron irradiation of the polycrystalline Fe, marked as F02, was carried out in the BOR-60 facility in Russia, which is a fast, sodium-cooled reactor operating on either $90 \%$ enriched uranium oxide or mixture of up to $40 \%$ plutonium oxide. F02 was irradiated at $386^{\circ} \mathrm{C}$ to a radiation dose of $16.6 \mathrm{dpa}$ under a fast neutron flux of $1.64 \times 10^{19}$ $\mathrm{n} / \mathrm{m}^{2} / \mathrm{s}(\mathrm{E}>0.1 \mathrm{MeV})$ corresponding to an appropriate damage rate of $8.8 \times 10^{-7} \mathrm{dpa} / \mathrm{s}$.

The helium implantation was conducted at room temperature with an ion flux of $4 \times 10^{15}$ $\mathrm{He} / \mathrm{m}^{2} / \mathrm{s}$ using an ion gun installed on the ORNL Gas Implantation and Thermal Desorption System. The samples were electropolished with the Struers ${ }^{\circledR}$ electrolyte A2 prior to He implantation. $10 \mathrm{keV}$ He implantation was performed on both the reference and as-irradiated (A4 and F02, respectively) samples to a fluence of $7 \times 10^{18} \mathrm{He} / \mathrm{m}^{2}$ under a vacuum of $9 \times 10^{-8}$ torr. Probabilistic depth distributions of generated Frenkel pairs and stopped He ions during implantation in iron were evaluated using SRIM2013 [37] in the mode of ion distribution and quick calculation of damage. For the $10 \mathrm{keV} \mathrm{He}$ implantation, the maximum production of Frenkel pairs by the $10 \mathrm{keV} \mathrm{He}$ in Fe occurs about $26 \mathrm{~nm}$ below the implantation surface, while the peak in the He distribution occurs at $50 \mathrm{~nm}$. On average, 33 Frenkel pairs are generated per He ion in average, corresponding to a peak displacement damage of $0.05 \mathrm{dpa}$ at an implantation fluence of 
$7 \times 10^{18} \mathrm{He} / \mathrm{m}^{2}$. It is important to note that room temperature He implantation into $\mathrm{Fe}$ at the relatively low fluence of $7 \times 10^{18} \mathrm{He} / \mathrm{m}^{2}$ has not been previously observed to produce He bubbles, as a result of the low thermal mobility of He and vacancy at room temperature, in addition to the low implanted He concentration [38] [39].

Following the He implantation, the implanted samples were transported under vacuum $\left(\sim 10^{-8}\right.$ torr $)$ to the thermal desorption chamber by a magnetic controlled linear manipulator, in order to avoid any further surface oxidation. The sample was then heated using a customized electrical resistivity heater to $1100^{\circ} \mathrm{C}$ with a linear ramping rate of $0.5^{\circ} \mathrm{C} / \mathrm{s}$. The released He was captured by a quadruple mass spectrometer and calibrated by using a Vacuum Technology Incorporated (VTI) standard He leak. The ultra-high vacuum of the thermal desorption chamber, $\sim 1 \times 10^{-9}$ torr, provides a very low $\mathrm{He}$ background at a level of $2 \times 10^{10} \mathrm{He} / \mathrm{s}$, as recorded by the mass spectrometer, during the heating process.

Microstructure characterization was performed on the neutron-irradiated Fe samples before and after TDS measurements in the low activation materials development and analysis lab (LAMDA) at ORNL with a JEOL JEM 2100F field emission gun scanning transmission electron microscope (FEG-(S)TEM) operating at 200kV. Electron transparent TEM foils were prepared using a focused ion beam system (FEI Quanta 3D Dual Beam) operated at $30 \mathrm{kV}$ for initial milling and at $5 \mathrm{kV}$ for final thinning to minimize the FIB-induced artifacts. All samples underwent an additional $900 \mathrm{eV}$ Ar ion mill using a Fischione Model 1040 NanoMill to reduce sample thickness and provide final cleaning of the sample prior to microstructural investigations.

\section{Results and discussion}

\subsection{TDS of reference samples}

Figure 1 shows a comparison of the thermal He desorption spectra from the reference polycrystalline and single crystalline Fe samples implanted with $10 \mathrm{keV} \mathrm{He}$ ions to a fluence of $7 \times 10^{18} \mathrm{He} / \mathrm{m}^{2}$, during thermal annealing with a constant temperature ramping rate of $0.5^{\circ} \mathrm{C} / \mathrm{s}$. An energy scale is included on the top axis (abscissa), in correlation with the temperature scale presented on the bottom axis. A simple first order dissociation kinetic model was used to calculate the activation energies corresponding to the various He desorption peaks and associate desorption events to mechanisms occurring at atomistic scales. Assuming He desorption follows a first order kinetic dissociation model, the evolution of the remaining He content (number density) in Fe can be expressed by

$$
\frac{d N}{d t}=-N f \exp \left(-\frac{E}{k_{B} T}\right)
$$

where $\mathrm{N}$ is the number density of remaining He atoms in the sample corresponding to a given desorption event, $\mathrm{f}$ is the jump frequency (assumed to be $1 \times 10^{13} / \mathrm{s}$ ), $\mathrm{E}$ is the activation energy of dissociation frequency, $\mathrm{k}_{\mathrm{B}}$ is the Boltzmann constant, and $\mathrm{T}$ is the 
temperature associated with the desorption peak. By solving $\frac{d^{2} N}{d t^{2}}=0$ with a linear temperature ramp (i.e., $\frac{d T}{d t}=\beta$ ), an expression is obtained to calculate the activation energy corresponding to each He desorption peak, in the form of

$$
\ln \left(\beta / T^{2}\right)=-\frac{E}{k_{B} T}+\ln \left(\frac{f k_{B}}{E}\right)
$$

It is noted that this model assumes that there are no mutual transformations among trapping sites. This is not likely to match reality, since He experiences a complex evolution including multiple capture and dissociation processes of various clusters.

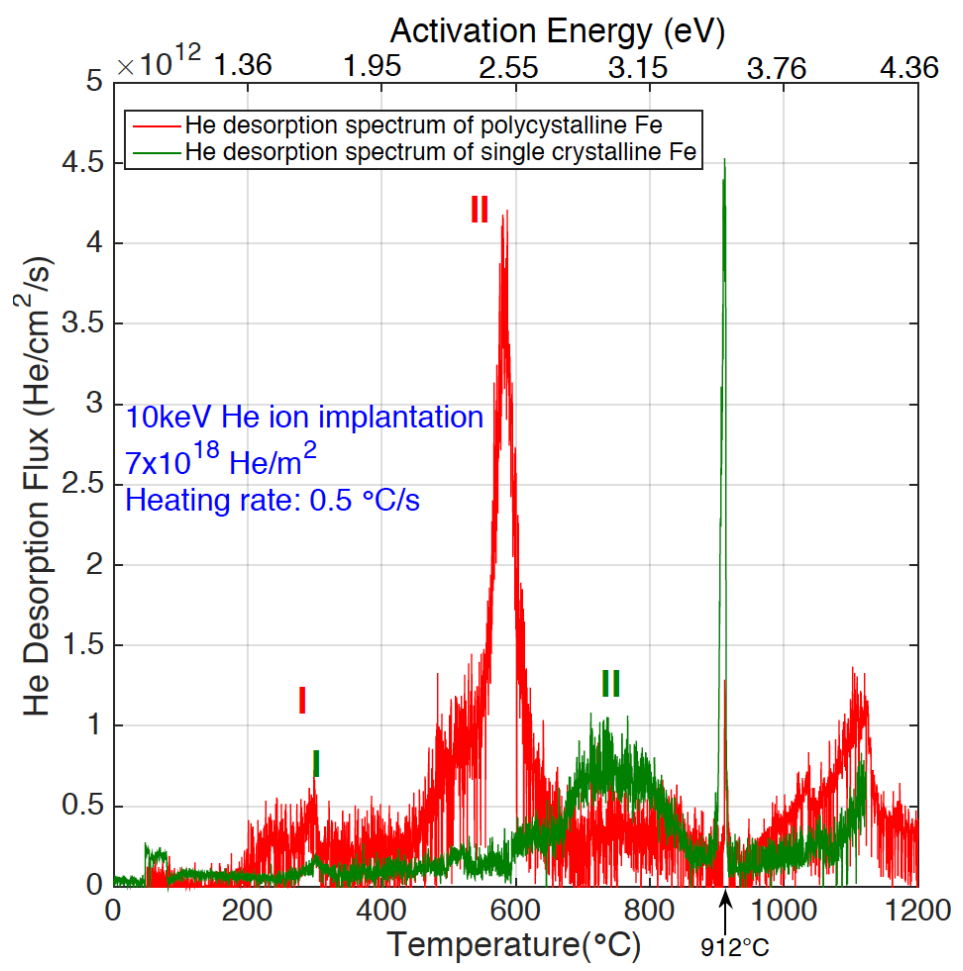

Figure 1. Thermal helium desorption spectra of the non-irradiated, high purity polycrystalline $\mathrm{Fe}$ (Red) and high purity single crystalline $\mathrm{Fe}$ (Green) following $10 \mathrm{keV}$ He implantation.

Both He desorption spectra show a sharp release peak at $912^{\circ} \mathrm{C}$, indicating the alphagamma phase transformation of Fe, which was used to calibrate the measured sample temperature. The measured He desorption spectra can be divided into two groups within the temperature range below $912^{\circ} \mathrm{C}$. Group I lies on the low temperature side, spanning from room temperature up to $\sim 350^{\circ} \mathrm{C}$, and is the same for both samples although more $\mathrm{He}$ is released from the polycrystalline Fe sample in Group I. The second desorption peaks for the polycrystalline Fe specimen (Group II) occurs from $450^{\circ} \mathrm{C}$ to $800^{\circ} \mathrm{C}$. However, in the single crystalline Fe specimen, Group II shifts to a higher temperature from $600^{\circ} \mathrm{C}$ to $900^{\circ} \mathrm{C}$. Both spectra showed He desorption peaks in the FCC temperature region $\left(>912^{\circ} \mathrm{C}\right)$. The difference in the observed Group II of these two samples is rationalized by 
the existence of grain boundaries providing relatively weakly bound trapping sites, and/or quick pathways for He transport, in polycrystalline Fe as well as a difference in impurities impacting the energetics of He-defect interactions [27] [40].

In Ref. [28] [41], the combination of a spatially-dependent cluster dynamics model based on diffusion-reaction rate theory and THDS measurements enabled the identification of the dominant He-vacancy clusters, from which the He is dissociated contributing to the measured He desorption peaks. In general, Group I can be ascribed to He that dissociates from small, helium-rich vacancy clusters, e.g., $\mathrm{He}_{2} \mathrm{~V}$, which is formed during the $\mathrm{He}$ implantation process and is readily dissociated during the low temperature annealing. In addition, the He atoms attached to pre-existing line dislocations or dislocation loops could also possibly dissociate in this low temperature region and contribute to the observed weak He desorption peaks [6, 36]. Group II is generally attributed to He dissociation from large He-V clusters formed during the annealing process as well as stable, small He-V clusters, e.g., $\mathrm{HeV}$ with a binding energy in the range from 2.3 to 3.7 $\mathrm{eV}$, as determined by different modeling methods [5] [11, 13] [14, 42] [43]. It is emphasized that the He desorption peak can not generally be interpreted to occur from only the dissociation of one type of cluster, but rather results from the collective behavior governing the evolution of all He-V clusters present within the material. In addition, it has been previously observed that interstitial impurities (i.e., C, N, O, etc.) impact the energetics of He-V clusters and significantly promote a shift of the measured $\mathrm{He}$ desorption peaks [40] [28, 44]. In terms of the overall helium inventory, the total desorbed He during the temperature ramping process are $86 \%$ and $81 \%$ of the initially implanted He quantity in polycrystalline and single crystalline Fe samples, respectively, which implies that some larger $\mathrm{He}_{\mathrm{m}} \mathrm{V}_{\mathrm{n}}$ clusters were formed and were sufficiently thermally stable to survive the high temperature annealing process. 


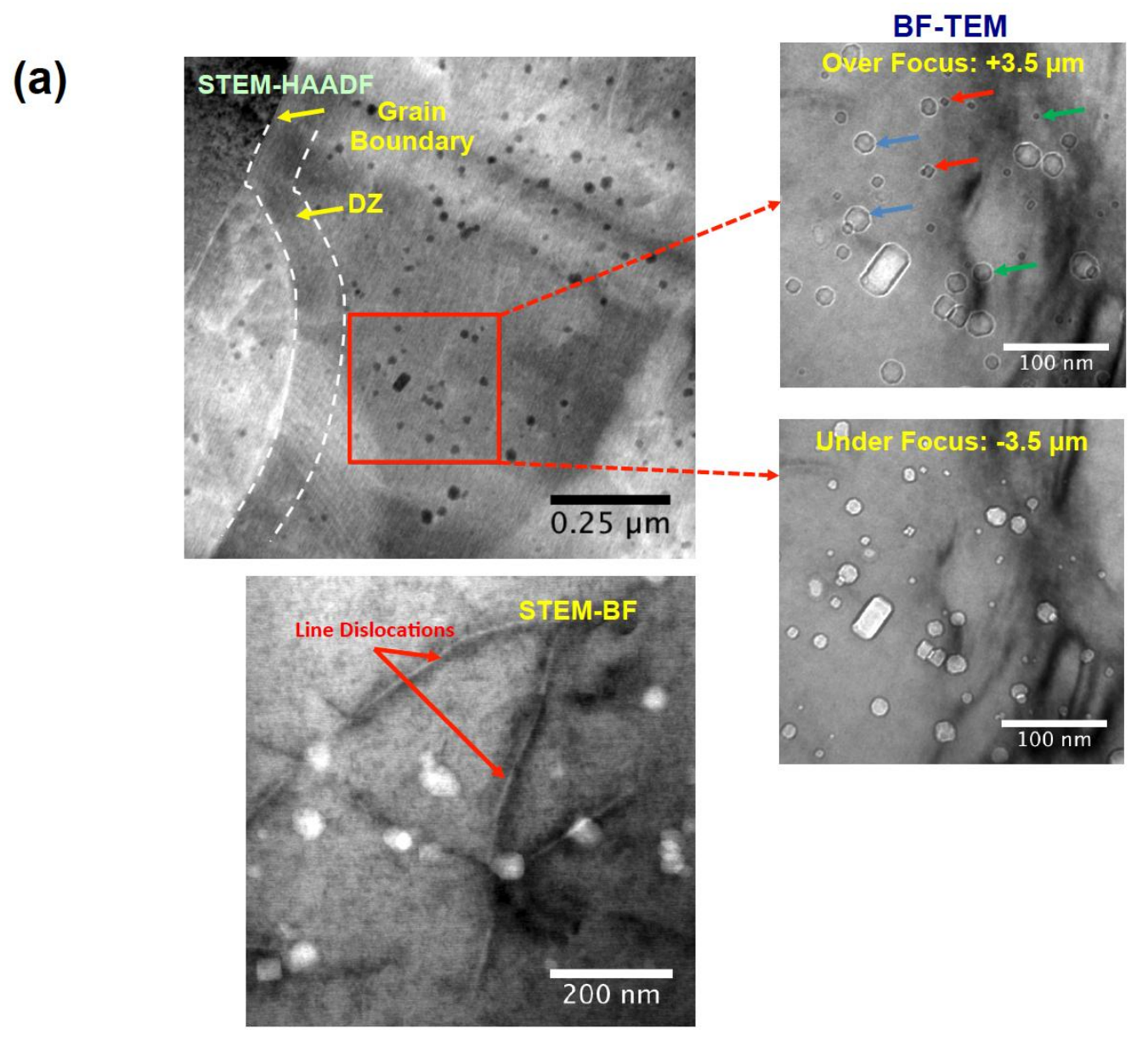


(b)
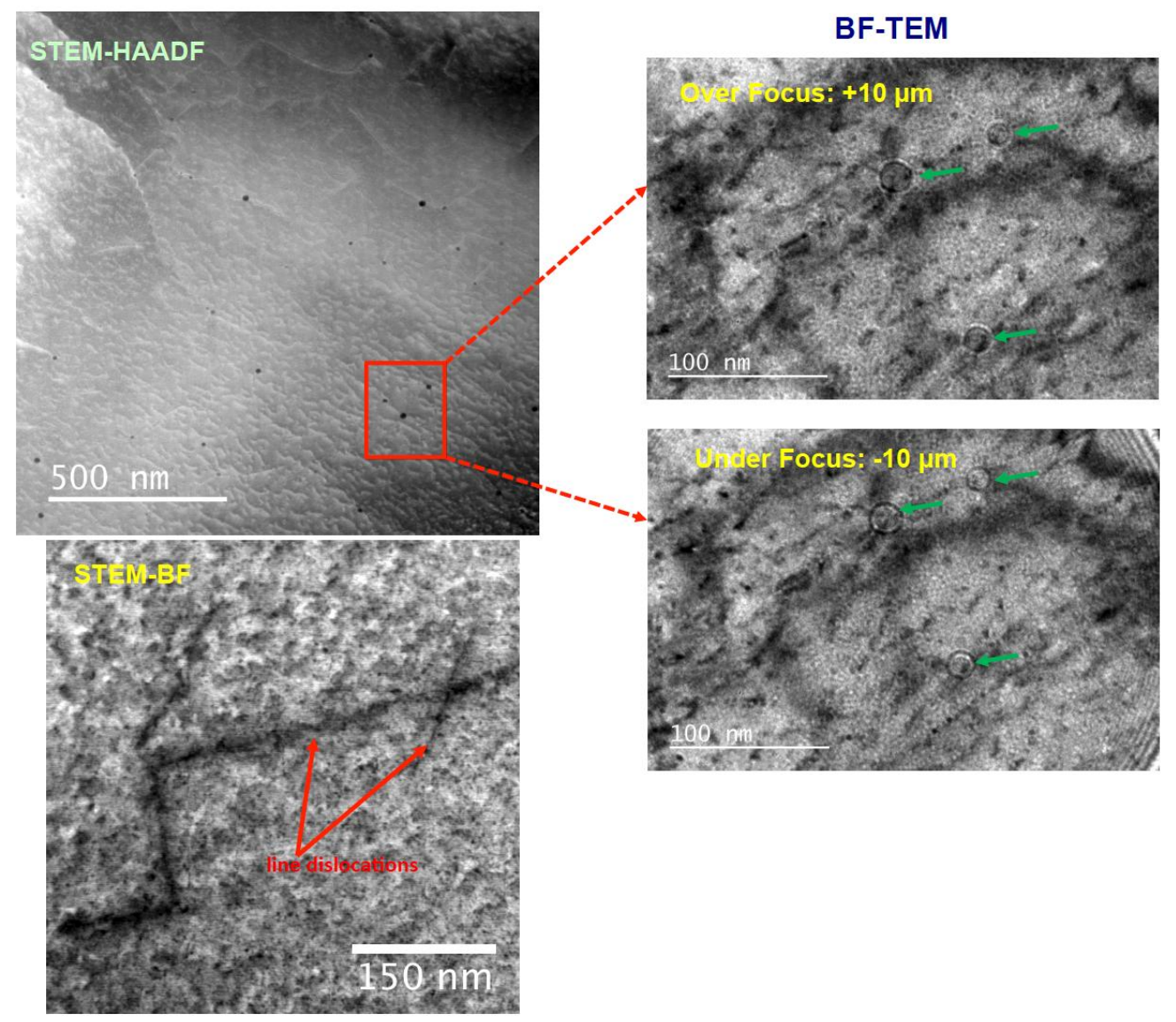

Figure 2. High-angle annular dark field (HAADF) and STEM bright field imaging of (a) as-irradiated polycrystalline $\mathrm{Fe}$ specimen, $\mathrm{F} 02$, irradiated in $\mathrm{BOR}-60$ to 16.6 dpa at $386^{\circ} \mathrm{C}$; (b) as-irradiated single crystalline Fe specimen, A4, irradiated in HFIR to 5 dpa at $300^{\circ} \mathrm{C}$. The two inlets on the right of (a) and (b) show the bright field TEM images of the marked region in the over focus and under focus conditions.

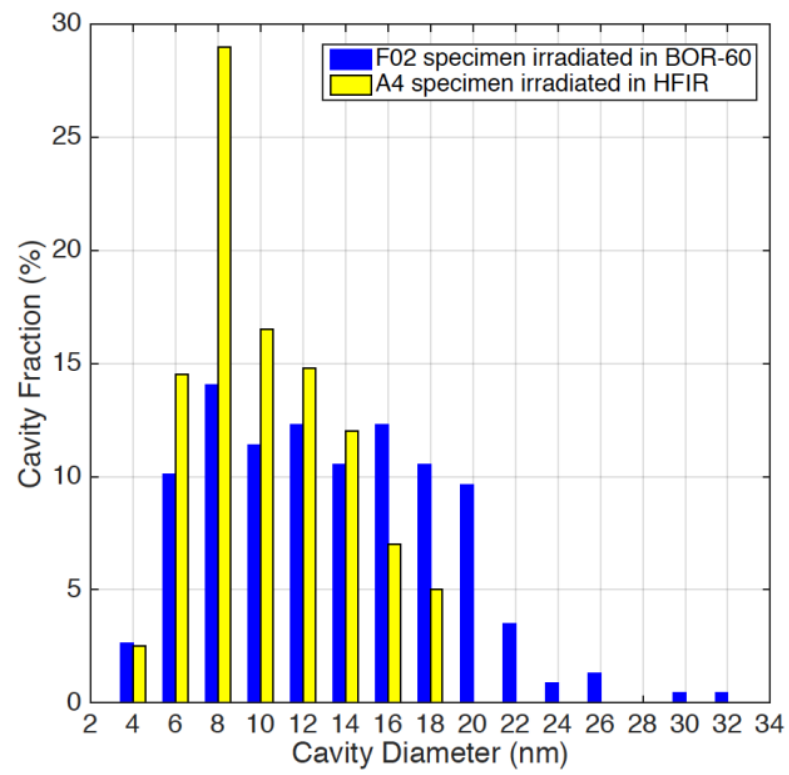


Figure 3. Cavity size distribution in $\mathrm{F} 02$ specimen irradiated at $386^{\circ} \mathrm{C}$ to 16.6 dpa in BOR-60 and A4 specimen irradiated at $300^{\circ} \mathrm{C}$ to $5 \mathrm{dpa}$.

\subsection{Microstructure of neutron-irradiated Fe samples}

TEM observations were performed to characterize the Fe microstructures following neutron irradiation, in order to identify the possible defect trapping features during the subsequent He ion implantation and desorption measurements. Figure 2 (a) shows the high angle annular dark field (HAADF) imaging of F02, irradiated to $16.6 \mathrm{dpa}$ at $386^{\circ} \mathrm{C}$ in BOR-60. It is evident that a high number density of cavities are dispersed within the grains, having an approximately continuous size distribution. However, no large dislocation loops were observed except a relatively low number density of line dislocations, which had limited association with the observed cavities. A denuded zone with a width of 80 to $120 \mathrm{~nm}$ was observed in the vicinity of grain boundaries, indicating that these grain boundaries are strong defect sinks. The observed cavities appear as squares (red arrows), truncated squares/rectangles (blue arrows), or circles (green arrows). The size distribution of cavities counted in a total area of $1.466 \mu \mathrm{m}^{2}$ of F02 specimen is shown in Figure 3. The resulting number density of the counted cavities is $\sim 1 \times 10^{21} \mathrm{~m}^{-3}$ and the average diameter is $12.3 \mathrm{~nm}$. In comparison, the visible cavities in the A4 specimen following neutron irradiation in HFIR at $300^{\circ} \mathrm{C}$ to $5 \mathrm{dpa}$ are sparsely distributed within the grains with a lower number density of $\sim 8 \times 10^{19} \mathrm{~m}^{-3}$ and an average diameter of $9.0 \mathrm{~nm}$, as shown in Fig. 2 (b) and Fig. 3, respectively. The lower number density and smaller cavity size observed in A4 (irradiated in HFIR) are ascribed to the lower irradiation temperature and radiation dose in comparison with sample F02 (irradiated in BOR-60). Moreover, line dislocations were also observed, similar to the F02 sample. In addition to the visible cavities observed by TEM observations, it is expected that a large amount of smaller, sub-visible vacancy clusters exist within these neutron-irradiated Fe samples.

The other outstanding phenomenon is that transmutant He is produced in iron by neutron irradiation in these two reactors due to $(n, \alpha)$ nuclear reactions. For example, neutron exposure in BOR-60 produces 0.2 appm He/dpa in Fe, equivalent to a total $3.3 \mathrm{appm}$ transmutant He uniformly generated within the as-irradiated F02. The transmutant He production rate in HFIR (single crystalline Fe) is nearly as large, with an estimated production level that is $72 \%$ of that of the BOR-60 irradiation, or about $0.14 \mathrm{appm} / \mathrm{dpa}$ [45]. Therefore, 0.72 appm He is expected within the as-irradiated A4. The reactorproduced transmutant He could diffuse rapidly to the surfaces of the Fe samples at 300 and $386^{\circ} \mathrm{C}$ and desorb, due to its low migration energy of $0.06 \mathrm{eV}$ [42]. Of course, however, the continuous neutron irradiation produces a large concentration of defects that serve as trapping sites, and thus likely result in the trapping of transmutant He within these defects, especially vacancy clusters. Correspondingly, it is likely that the majority of the transmuted He atoms exist as He-vacancy clusters, cavities containing $\mathrm{He}$ or $\mathrm{He}-$ dislocation complexes due to the extremely low solubility of He in Fe. TEM analysis indicated that a relatively large number density of cavities was formed in the as-irradiated F02 and A4 samples (Fig. 2 (a)). The presence of He is expected to stabilize cavity nucleation, such that many of these cavities, especially at smaller sizes, are actually $\mathrm{He}$ bubbles. Note that for consistency, we will use the term of 'cavities' to represent the 
TEM-visible open volume defect features throughout the paper since the helium gas pressure (He/V ratio) is unknown.

\subsection{TDS of He-implanted Fe samples subject to neutron irradiation}
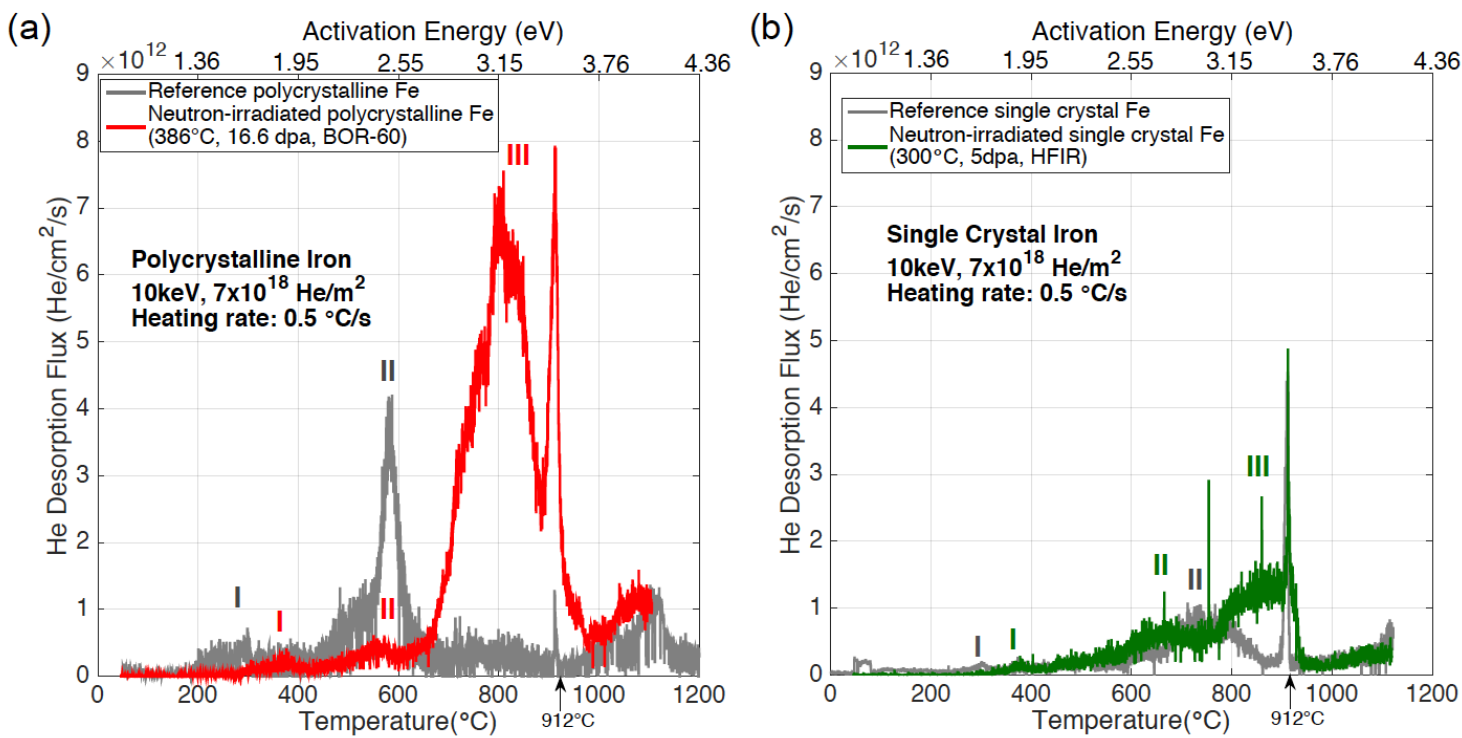

Figure 4. Comparison of He desorption spectra of the He-implanted samples before and after neutron irradiations: (a) polycrystalline $\mathrm{Fe}$; (b) single crystalline Fe.

Following neutron irradiation, $10 \mathrm{keV} \mathrm{He}$ ion implantation at room temperature was performed on the two as-irradiated samples, F02 and A4, respectively, prior to the TDS measurement. In order to compare with the un-irradiated samples, the same implanted $\mathrm{He}$ fluence of $7 \times 10^{18} \mathrm{He} / \mathrm{m}^{2}$ was applied. Figure 4 shows the comparison of the measured $\mathrm{He}$ desorption spectra of these Fe samples with and without neutron irradiation, respectively. It is immediately apparent that much more helium was thermally desorbed from the neutron irradiated specimen F02 compared to A4. The sharp He release peak at $912{ }^{\circ} \mathrm{C}$, indicating the BCC-FCC phase transformation, still appears in the He desorption spectra of both samples, and again was used for temperature calibration. The largest amount of He desorption (denoted as Group III) in each irradiated sample was observed in a new, higher temperature region relative to the un-irradiated specimens, and thus three major He release groups were observed in the $\mathrm{BCC}$ region of the He desorption spectra of these neutron irradiated samples. The He desorption behavior in the FCC regime of the neutron irradiated samples are quite similar to those of un-irradiated samples, having a $\mathrm{He}$ desorption peak at $\sim 150^{\circ} \mathrm{C}$, although some of the spectra were not completely developed.

For the polycrystalline Fe sample, F02, the major He desorption peak shifted to a higher temperature region of $650 \sim 900^{\circ} \mathrm{C}$ with a peak occurring at $800^{\circ} \mathrm{C}$. The peak of Group I shifted up in temperature region from $290^{\circ} \mathrm{C}$ to $360^{\circ} \mathrm{C}$, while the Group II desorption regime can still be identified in essentially the same temperature range, albeit with a much weaker intensity. In contrast to the un-irradiated sample, in which the major 
trapping sites for He prior to TDS were assumed to be single vacancy and small vacancy clusters (smaller than $\mathrm{V}_{8}$ [28]) produced during the $10 \mathrm{keV} \mathrm{He}$ implantation, the intense neutron irradiation exposure in BOR-60 gave rise to the production of a significant number density of large vacancy clusters and cavities as well as line dislocations, as shown in Section 3.2, which were assumed to be trapping sites for the newly implanted He. Therefore, the observed He desorption peaks can be ascribed to the significant evolution of both cavities and large He-V clusters during the TDS annealing process. The resultant stable large $\mathrm{He}-\mathrm{V}$ clusters or cavities following He implantation can only be dissociated when the temperature is sufficiently high. The largest He release peak around $800^{\circ} \mathrm{C}$ corresponds to an activation energy of $3.15 \mathrm{eV}$, consistent with He-V clusters (possibly including bubbles) having a He-to-V ratio of $~ 3.0$ [5]. In addition, dislocations are expected to bind He atoms. Atomistic simulations [46] [6] indicate that the dissociation energy of He from dislocation loops are in the range from 1.4 to $2.3 \mathrm{eV}$, dependent on the dislocation type and orientation. Therefore, He detrapping from dislocations could also contribute to the observed Group I and II desorption peaks.

For the single crystalline Fe specimen, A4, a new He desorption group was also observed in the temperature region from $750^{\circ} \mathrm{C}$ to the phase transformation point at $912^{\circ} \mathrm{C}$, which is assumed to result from He dissolution from large $\mathrm{He}-\mathrm{V}$ clusters or cavities containing He. Such cavities are presumably produced by either the combination of implanted He and pre-existing large vacancy clusters or cavities generated by neutron irradiation in HFIR, or the thermally-driven growth process of cavities containing He during the TDS measurements. The two major He desorption peaks are observed at temperatures of $666^{\circ} \mathrm{C}$ and $857^{\circ} \mathrm{C}$, corresponding to activation energies of $2.75 \mathrm{eV}$ (small He-V clusters like $\mathrm{He}_{2} \mathrm{~V}_{2}$, or larger $\mathrm{He}_{\mathrm{m}} \mathrm{V}_{\mathrm{n}}$ clusters/bubbles with a He/V ratio of $\sim 4$ ) and $3.32 \mathrm{eV}$ (large $\mathrm{He}_{\mathrm{m}} \mathrm{V}_{\mathrm{n}}$ clusters/bubbles with $\mathrm{He} / \mathrm{V}$ ratio of $~ 2.8$ ), respectively. The Group I He desorption peak shifted from 295 to $373^{\circ} \mathrm{C}$, consistent with the shift observed in F02, implying a similar He dissociation mechanism, which is most likely due to the He detrapping from unstable cavities containing He, larger He-V clusters compared to the He-implanted reference samples, or He-dislocation complexes.

Table 1. Helium content in F02 and A4, as a function of condition.

\begin{tabular}{lllllll}
$\begin{array}{l}\text { Sample } \\
\text { ID }\end{array}$ & $\begin{array}{l}\text { Surface } \\
\text { area } \\
\left(\mathrm{mm}^{2}\right)\end{array}$ & $\begin{array}{l}\text { Thickness } \\
(\mathrm{mm})\end{array}$ & $\begin{array}{l}\text { Transmutant } \\
\text { He during } \\
\text { neutron } \\
\text { irradiation } \\
(\text { appm })\end{array}$ & $\begin{array}{l}\text { Implanted } \\
\text { amount } \\
(\text { appm }) *\end{array}$ & $\begin{array}{l}\text { Total He } \\
\text { amount prior } \\
\text { to TDS } \\
\text { measurement } \\
\text { (appm) }\end{array}$ & $\begin{array}{l}\text { Desorbed He } \\
\text { amount } \\
\text { during TDS } \\
\text { measurements } \\
(\text { appm) }\end{array}$ \\
\hline F02 & 7.0 & 0.25 & 3.32 & 0.56 & 3.88 & 2.76 \\
A4 & 16 & 0.5 & 0.72 & 0.12 & 0.84 & 0.11 \\
\hline
\end{tabular}

*In order to simplify the He concentration calculation, the implanted He is assumed to distribute throughout the whole sample homogeneously. Although the same amount of He was implanted, the 'average' He concentration is different due to the different sample sizes in these two samples.

As indicated by Figure 4, it is clearly apparent that the total desorbed $\mathrm{He}$ from the neutron irradiated samples is more than that from the un-irradiated samples. This is 
rationalized by the fact that transmutant $\mathrm{He}$ due to $(n, \alpha)$ nuclear reactions was produced when Fe was exposed to neutron irradiation, as mentioned in Section 3.2. Table 1 lists the He content in these samples as a function of the specimen condition. Considering the subsequent He ion implantation at room temperature following neutron irradiation, the total He contained in F02 and A4 is approximately 3.88 and 0.84 appm, respectively. It is important to note that these values were simply obtained by dividing the sum of the transmutant and implanted He amounts by the total atom number in each sample. The integrated concentration of desorbed He from the neutron irradiated polycrystalline and single crystalline Fe during the THDS measurements are equivalent to 2.76 and 0.11 appm He, respectively. In other words, $72 \%$ of the initial He was released from F02, while only $13 \%$ of the He contained in A4 was desorbed. The remaining He is expected to exist in the form of stable He vacancy clusters or cavities, as would be expected based on surviving the thermal annealing up to about $1150^{\circ} \mathrm{C}$ during the TDS measurements.

Based on the assessment of thermal stability of He-vacancy clusters in iron [5] [47], the $\mathrm{He}-\mathrm{V}$ clusters or cavities containing $\mathrm{He}$ must have a $\mathrm{He} / \mathrm{V}$ ratio of $\sim 2.0$ to survive the annealing process. Although the energetics database of He- $\mathrm{V}$ clusters in FCC iron is not available, it is reasonable to assume that the stability of He bubbles in FCC temperature region still depends on the ratio of $\mathrm{He} / \mathrm{V}$, which has been verified by the simulation of energetics of He-V clusters in FCC Ni [48]. Therefore, it is deduced that there are more visible cavities containing He in the single crystalline, HFIR irradiated A4 sample following the TDS measurement compared to that of F02, and these features may have been produced either due to the growth or coarsening of large, but sub-visible He-V clusters or directly during neutron irradiation such that they are sufficiently thermally stable to survive the annealing during the TDS measurement. In contrast, the He-V clusters or cavities containing $\mathrm{He}$ in $\mathrm{F} 02$ are less stable and most of them will dissolve with the release of He and vacancy. Thus, the number density of visible cavities in F02 should decrease after TDS measurement.

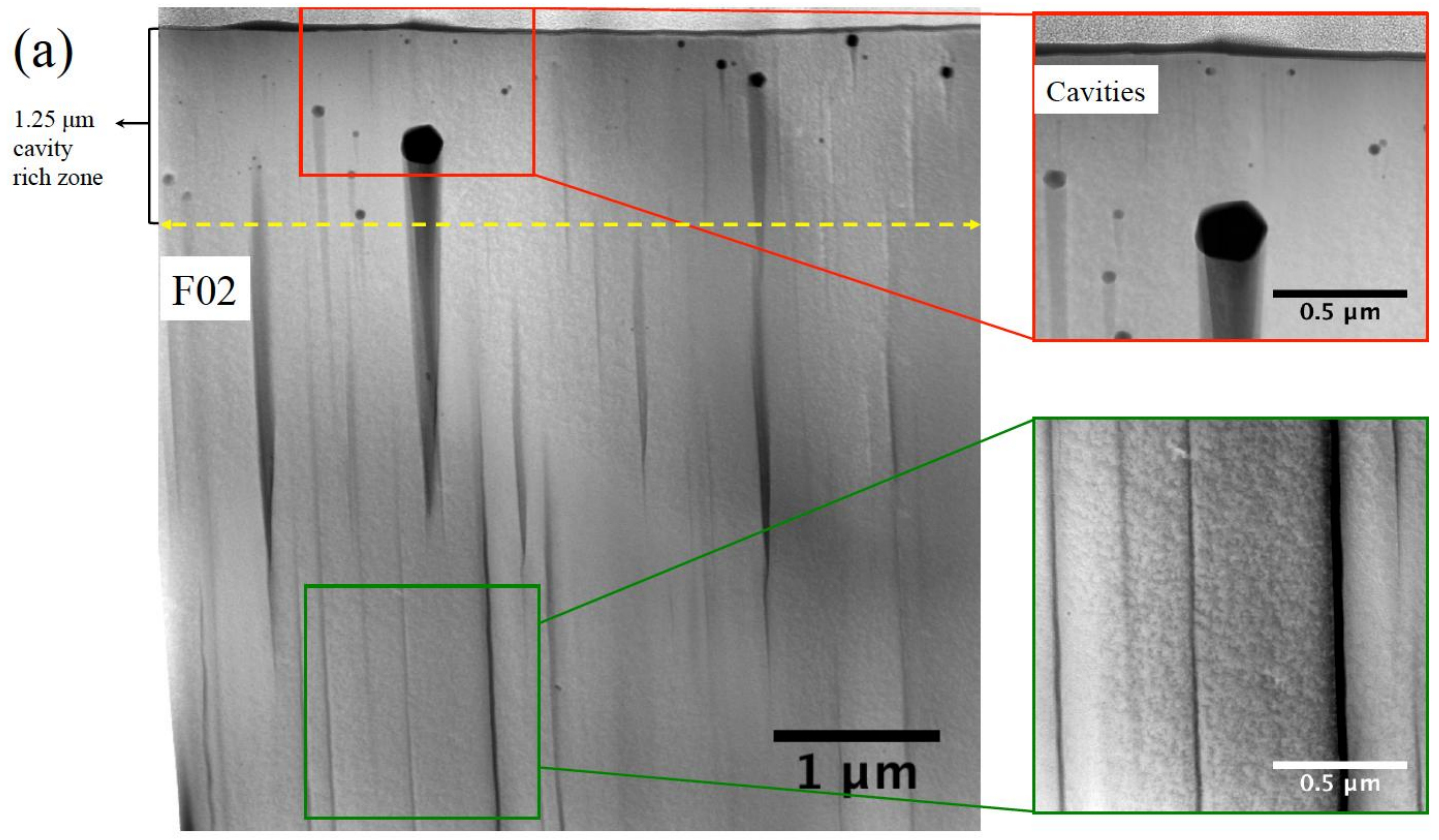




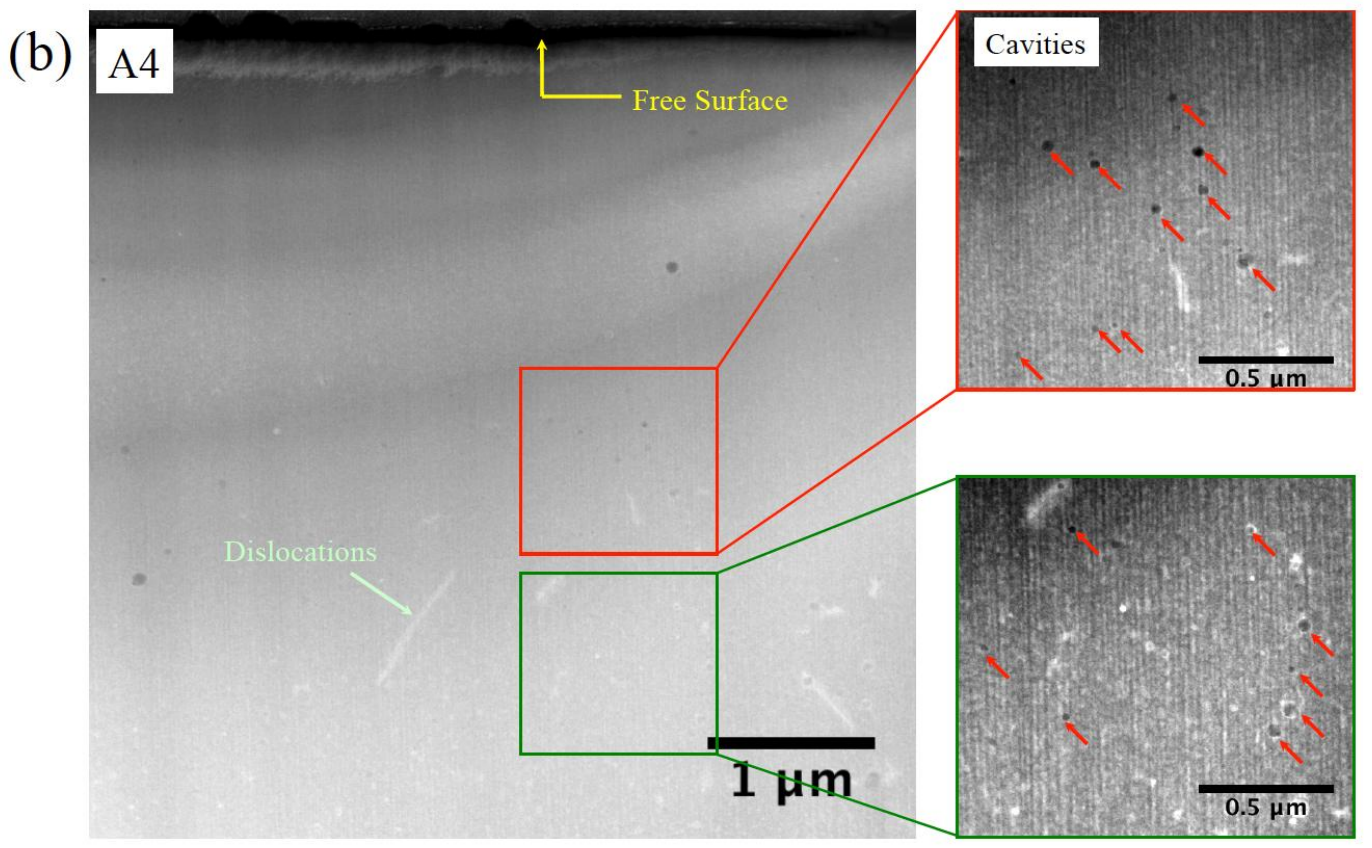

Figure 5. HAADF imaging of neutron irradiated (a) polycrystalline Fe specimen, F02 and (b) single crystal Fe specimen, A4, following TDS measurements.

\subsection{Microstructure of neutron-irradiated Fe samples post TDS measurement}

In order to validate this hypothesis, TEM observations on these two samples following TDS measurements were performed, as shown in Figure 5. For F02, it is obvious that significant microstructural evolution occurred during the thermal annealing process. The number density of the visible cavities decreased remarkably compared to the as-irradiated condition, from $1 \times 10^{21} \mathrm{~m}^{-3}$ to $4.3 \times 10^{19} \mathrm{~m}^{-3}$, while the average size increased from 12.3 $\mathrm{nm}$ to $24.3 \mathrm{~nm}$. The other clear observation is that the remaining cavities were found in an area relatively close to the free surface $(<1.25 \mu \mathrm{m}$ depth), as shown in Figure 5 (a). Most of the cavities are faceted without association of any other microstructural defects. However, the microstructure of He-implanted A4 following TDS is completely different from that of F02, as shown in Fig. 5 (b). Cavities were observed throughout the studied area. No obvious spatial dependence was observed. The average number density of the visible cavities is $4.5 \times 10^{19} \mathrm{~m}^{-3}$ with an average size of $28 \mathrm{~nm}$. The values for these two parameters in the as-irradiated sample are $8 \times 10^{19} \mathrm{~m}^{-3}$ and $9.0 \mathrm{~nm}$, respectively, and thus, there was some coarsening of the cavity population during the TDS process but not nearly as dramatic as in specimen F02. Figure 6 shows the cavity size distribution of F02 and A4 before and after TDS measurements, as determined from TEM analysis. Significant coarsening of cavities was observed and the size distributions of these two samples after TDS measurements were much broader in comparison with the asirradiated state, implying the TDS thermal annealing drove significant growth of cavities for both samples through absorbing free He and V dissociated from unstable He- $\mathrm{V}$ clusters or cavities containing He, albeit the cavity rich zone was only found in the near surface area for F02. 

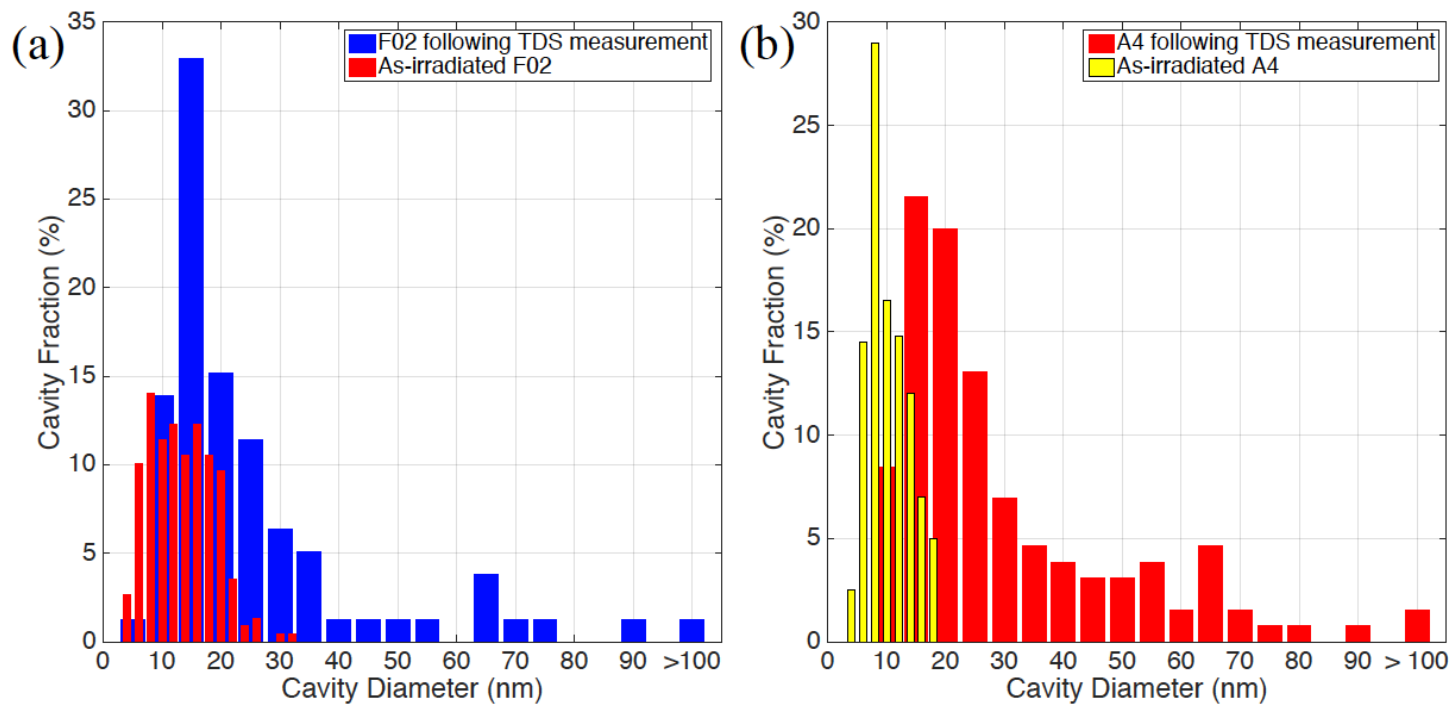

Figure 6. Cavity size distribution in (a) F02 specimen irradiated at $386^{\circ} \mathrm{C}$ to 16.6 dpa in BOR-60 and (b) A4 specimen irradiated at $300^{\circ} \mathrm{C}$ to 5 dpa following the TDS measurements. The cavity distribution of asirradiated conditions was also included for comparison.

\subsection{Discussion}

The significantly different cavity evolution pathways during the TDS thermal annealing in these two samples are believed to result from the difference in the initial microstructures prior to $10 \mathrm{keV} \mathrm{He}$ implantation and the subsequent TDS measurement, along with differences in the thermal stability of the pre-existing cavities (likely containing different He pressures). If we make a simple assumption that all the transmutation-produced He was contained in the TEM-visible cavities (shown in Figure 2) after neutron irradiation (of course, this is not the reality since He is likely also trapped at other damage features); this simple assumption provides a basic idea of the average $\mathrm{He} / \mathrm{V}$ ratio in the visible cavities of the two neutron irradiated samples. The average $\mathrm{He} / \mathrm{V}$ ratio of the cavities in F02 is only $14 \%$ of that in A4, based on simply partitioning the transmutant He into the number density and size of the cavities in these two samples. Therefore, one would expect that the cavities in F02 are much less stable to vacancy dissolution (and eventually He release) during the TDS annealing process, in comparison with those in A4. This might result in the significant He release from F02 during the TDS thermal annealing process and the different cavity evolution observed in Figures 5 and 6.

The observation of the cavity rich zone near the free surface in F02 could be rationalized by a mechanism wherein both implanted He and dissociated He from the bulk cavities promoted the nucleation and growth of the cavities (likely containing different He pressures) near surface during the TDS annealing process. The post-irradiation He implantation introduces a large concentration of He in a shallow area, i.e., $140 \mathrm{~nm}$ beneath the free surface based on SRIM calculation. This implanted He can readily react with vacancies, vacancy clusters or cavities, as well as line dislocations or dislocation loops. During the TDS thermal anneal with a ramp rate of $0.5^{\circ} \mathrm{C} / \mathrm{s}$, it is reasonable to 
expect some fraction of the implanted He to be released from relatively weak trapping sites (e.g., small vacancy clusters, over-pressurized He bubbles, and dislocation loops). It is also reasonable to expect some fraction of this now-mobile He to diffuse deeper below the surface and then be captured by the vacancy clusters or under-pressurized cavities located deeper below the surface. The TDS measurements indicated that $72 \%$ of preexisting He was desorbed from specimen F02. Therefore, a large flux of transmutant $\mathrm{He}$ dissociated from damage features was expected to diffuse from bulk to the free surface, a strong defect sink. A fraction of these He could be captured by the cavities or other defects on their way to the free surface, contributing to the coarsening of cavities observed in the near surface. Although no visible cavities were observed in the bulk of F02 following TDS measurements, stable large He-V clusters or TEM-invisible He bubbles may exist throughout the bulk, given the significantly high activation energy of large stable He-V clusters or stable bubbles (> $4.0 \mathrm{eV}$ ) [5], which contribute to the $\mathrm{He}$ retention within F02 after TDS measurement.

In contrast, the TDS measurement indicated that $87 \%$ of initial He remained in A4 following the TDS measurement, as shown in Fig. 4 (b). TEM observation showed that a large number density of cavities survived the TDS annealing process. As discussed before, the average $\mathrm{He} / \mathrm{V}$ ratio of the cavities containing He in as-irradiated A4 is higher than that of F02, leading to more stable initial states of these cavities in A4. The relatively stable He cavities following neutron irradiation can then serve as precursors for the subsequent evolution during the He implantation and the annealing process and have a higher probability to survive the annealing process to produce a large number density of cavities, as observed in A4 following TDS, and shown in Fig. 5(b). Of course, significant evolution of cavities or $\mathrm{He}-\mathrm{V}$ clusters were expected due to the dissociation of $\mathrm{V}$ or $\mathrm{He}$ and capturing mobile species, which resulted in the growth of the survival cavities throughout the bulk of A4 following TDS measurement, as shown in Figure 5 (b) and Figure 6 (b).

Moreover, the comparison of TDS and TEM observations of these two samples also implied that more transmutant He was trapped by the local microstructural features (e.g., small vacancy clusters, impurities) in F02 without being captured by the large cavities during neutron irradiation, which could explain the significant desorption of He from this sample and reduced survival of cavities following TDS measurement. In single crystalline specimen A4, the majority of transmutant He was presumably trapped in the cavities, giving rise to the more stable cavities in the as-irradiated state. Therefore, more cavities survived the TDS annealing process and less He was released from the sample. We assert that the difference in the partition of transmutant He during neutron irradiation of these two samples might result from the different radiation damage rates of the two reactors as well as the impurity levels of the samples. However, more experiments are needed to confirm this hypothesis.

\section{Conclusion}

The neutron irradiation impact on thermal helium desorption behavior in high purity $\mathrm{Fe}$ were investigated by using THDS and TEM observations. The comparison of He 
desorption spectra from the reference and neutron-irradiated iron samples showed that the major He desorption peaks shift to a higher temperature and that a new desorption group was observed at higher temperatures in the neutron-irradiated samples due to the production of vacancy clusters or cavities, which can be strong trapping sites for He. Significant cavity evolution was expected during the thermal annealing processes of TDS measurements. Most of the cavities in the bulk of specimen F02 (BOR-60 irradiated polycrystalline $\mathrm{Fe}$ ) were dissolved while the remaining were observed only near the free surface. In contrast, the pre-existing cavities in sample A4 (HFIR irradiated single crystal) were more stable to survive the TDS annealing measurement. The post-TDS microstructures of neutron-irradiated Fe were heavily dependent on the initial $\mathrm{He} / \mathrm{V}$ ratio of the He-V clusters or cavities produced in the as-irradiated samples. The present study is expected to provide additional insights into the synergistic effects of neutron irradiation induced damage and the transmutant $\mathrm{He}$ in Fe-based alloys used for nuclear applications.

\section{Acknowledgments}

The work presented in this paper was partially supported by Laboratory Directed R\&D funds at ORNL. The research was also sponsored by the US Department of Energy Office of Fusion Energy Science under grant DE-AC05-00OR22725 with UT-Battelle LLC, and grant DOE-DE-SC0006661 with the University of Tennessee, Knoxville. The authors also acknowledge the IRP program led by Prof. Gary Was at University of Michigan for generously providing the iron specimen irradiated in BOR-60.

\section{Reference}

1. Zinkle, S.J. and G.S. Was, Materials challenges in nuclear energy. Acta Materialia, 2013. 61(3): p. 735-758.

2. Terrani, K.A., S.J. Zinkle, and L.L. Snead, Advanced oxidation-resistant ironbased alloys for LWR fuel cladding. Journal of Nuclear Materials, 2014. 448(1-3): p. 420-435.

3. Tan, L., L.L. Snead, and Y. Katoh, Development of new generation reduced activation ferritic-martensitic steels for advanced fusion reactors. Journal of Nuclear Materials, 2016. 478: p. 42-49.

4. Trinkaus, H., Energetics and formation kinetics of helium bubbles in metals. Radiation Effects and Defects in Solids, 1983. 78(1-4): p. 189-211.

5. Morishita, K., et al., Thermal stability of helium-vacancy clusters in iron. Nuclear Instruments and Methods in Physics Research Section B: Beam Interactions with Materials and Atoms, 2003. 202: p. 76-81.

6. Ventelon, L., B. Wirth, and C. Domain, Helium-self-interstitial atom interaction in $\alpha$-iron. Journal of Nuclear Materials, 2006. 351(1-3): p. 119-132.

7. Lucas, G. and R. Schäublin, Helium effects on displacement cascades in $\alpha$-iron. Journal of Physics: Condensed Matter, 2008. 20(41): p. 415206. 
8. Brimbal, D., et al., Dual-beam irradiation of $\alpha$-iron: Heterogeneous bubble formation on dislocation loops. Journal of Nuclear Materials, 2011. 418(1-3): p. 313-315.

9. Prokhodtseva, A., et al., Impact of He and Cr on defect accumulation in ionirradiated ultrahigh-purity $\mathrm{Fe}(\mathrm{Cr})$ alloys. Acta Materialia, 2013. 61(18): p. 69586971.

10. Schäublin, R., J. Henry, and Y. Dai, Helium and point defect accumulation: ( $i$ ) microstructure and mechanical behaviour. Comptes Rendus Physique, 2008. 9(34): p. 389-400.

11. Fu, C.-C., et al., Multiscale modelling of defect kinetics in irradiated iron. Nature Materials, 2004. 4(1): p. 68-74.

12. Trinkaus, H. and B.N. Singh, Helium accumulation in metals during irradiationwhere do we stand? Journal of Nuclear Materials, 2003. 323(2-3): p. 229-242.

13. Morishita, K., R. Sugano, and B.D. Wirth, MD and KMC modeling of the growth and shrinkage mechanisms of helium-vacancy clusters in Fe. Journal of Nuclear Materials, 2003. 323(2-3): p. 243-250.

14. Fu, C.-C. and F. Willaime, Ab initiostudy of helium in $\alpha-F e$ : Dissolution, migration, and clustering with vacancies. Physical Review B, 2005. 72(6).

15. Gao, N., et al., Formation of dislocation loops during He clustering in bcc Fe. J Phys Condens Matter, 2011. 23(44): p. 442201.

16. Kurtz, R.J. and H.L. Heinisch, The effects of grain boundary structure on binding of He in Fe. Journal of Nuclear Materials, 2004. 329-333: p. 1199-1203.

17. Heinisch, H.L., et al., Interaction of helium atoms with edge dislocations in $\alpha$ $F e$. Journal of Nuclear Materials, 2006. 351(1-3): p. 141-148.

18. Heinisch, H.L., F. Gao, and R.J. Kurtz, Atomistic modeling of helium interacting with screw dislocations in $\alpha$-Fe. Journal of Nuclear Materials, 2007. 367-370: p. 311-315.

19. $\mathrm{Xu}, \mathrm{D}$. and B.D. Wirth, Modeling spatially dependent kinetics of helium desorption in BCC iron following He ion implantation. Journal of Nuclear Materials, 2010. 403(1-3): p. 184-190.

20. Ortiz, C.J. and M.J. Caturla, Simulation of defect evolution in irradiated materials: Role of intracascade clustering and correlated recombination. Physical Review B, 2007. 75(18).

21. Samaras, M., Multiscale Modeling: the role of helium in iron. Materials Today, 2009. 12(11): p. 46-53.

22. Rothaut, J., H. Schroeder, and H. Ullmaier, The growth of helium bubbles in stainless steel at high temperatures. Philosophical Magazine A, 1983. 47(5): p. 781-795.

23. Lefaix-Jeuland, H., et al., Effect of grain microstructure on thermal helium desorption from pure iron. Journal of Nuclear Materials, 2013. 434(1-3): p. 152157.

24. Field, K.G., et al., Radiation tolerance of neutron-irradiated model $\mathrm{Fe}-\mathrm{Cr}-\mathrm{Al}$ alloys. Journal of Nuclear Materials, 2015. 465: p. 746-755.

25. Lefaix-Jeuland, H., et al., SIMS depth profiling of implanted helium in pure iron using $\mathrm{CsHe}+$ detection mode. Nuclear Instruments and Methods in Physics 
Research Section B: Beam Interactions with Materials and Atoms, 2013. 295: p. 69-71.

26. $\mathrm{Xu}, \mathrm{D}$., et al., Thermal helium desorption spectrometry of helium-implanted iron. Journal of Nuclear Materials, 2007. 367-370: p. 483-488.

27. $\mathrm{Xu}, \mathrm{D}$. and B.D. Wirth, Post-implantation thermal desorption of helium from poly- and single-crystalline iron. Journal of Nuclear Materials, 2009. 386-388: p. 395-399.

28. Hu, X., D. Xu, and B.D. Wirth, Quantifying He-point defect interactions in Fe through coordinated experimental and modeling studies of He-ion implanted single-crystal Fe. Journal of Nuclear Materials, 2013. 442(1-3): p. S649-S654.

29. Shiba, K. and A. Hishinuma, Low-temperature irradiation e ects on tensile and Charpy properties of low-activation ferritic steels. Journal of Nuclear Materials, 2000. 283-287: p. 474-477.

30. Klueh, R.L., et al., Mechanical properties of neutron-irradiated nickel-containing martensitic steels: II. Review and analysis of helium-effects studies. Journal of Nuclear Materials, 2006. 357(1-3): p. 169-182.

31. Klimenkov, M., A. Möslang, and E. Materna-Morris, Helium influence on the microstructure and swelling of $9 \%$ Cr ferritic steel after neutron irradiation to 16.3dpa. Journal of Nuclear Materials, 2014. 453(1-3): p. 54-59.

32. Zhang, H., et al., Irradiation hardening of ODS ferritic steels under helium implantation and heavy-ion irradiation. Journal of Nuclear Materials, 2014. 455(1-3): p. 349-353.

33. Parish, C.M., et al., Helium sequestration at nanoparticle-matrix interfaces in helium + heavy ion irradiated nanostructured ferritic alloys. Journal of Nuclear Materials, 2017. 483: p. 21-34.

34. Cao, X.Z., et al., Effects of dislocations on thermal helium desorption from nickel and iron. Journal of Nuclear Materials, 2011. 417(1-3): p. 1034-1037.

35. Sugano, R., et al., Microstructural evolution in $\mathrm{Fe}$ and $\mathrm{Fe}-\mathrm{Cr}$ model alloys after $\mathrm{He}+$ ion irradiations. Journal of Nuclear Materials, 2004. 329-333: p. 942-946.

36. Gong, Y.H., et al., Effect of dislocations on helium retention in deformed pure iron. Journal of Nuclear Materials, 2016. 482: p. 93-98.

37. Ziegler, J.F., M.D. Ziegler, and J.P. Biersack, SRIM - The stopping and range of ions in matter (2010). Nuclear Instruments and Methods in Physics Research Section B: Beam Interactions with Materials and Atoms, 2010. 268(11-12): p. 1818-1823.

38. Zelenskij, V.F., et al., Thermal desorption of helium from polycrystalline Ni irradiated to fluences ranging from 1 e17 to 1 e18 He/cm2. Journal of Nuclear Materials, 1987. 151: p. 22-33.

39. Chernov, I.I., et al., Gas porosity evolution and ion-implanted helium behavior in reactor ferritic/martensitic and austenitic steels. Journal of Nuclear Materials, 2015. 459: p. 259-264.

40. Ortiz, C.J., et al., Impurity effects on He diffusion in $\alpha$-Fe. Journal of Nuclear Materials, 2009. 386-388: p. 33-35.

41. $\mathrm{Xu}, \mathrm{D} ., \mathrm{X}$. Hu, and B.D. Wirth, A phase-cut method for multi-species kinetics: Sample application to nanoscale defect cluster evolution in alpha iron following helium ion implantation. Applied Physics Letters, 2013. 102(1): p. 011904. 
42. Ortiz, C.J., et al., He diffusion in irradiated $\alpha-F e$ : Anab-initio-based rate theory model. Physical Review B, 2007. 75(10).

43. Fu, C.-C. and F. Willaime, Interaction between helium and self-defects in $\alpha$-iron from first principles. Journal of Nuclear Materials, 2007. 367-370: p. 244-250.

44. Ortiz, C.J., et al., Influence of carbon on the kinetics of He migration and clustering in $\alpha$-Fefrom first principles. Physical Review B, 2009. 80(13).

45. Karasiov, A.V., Neutron flux spectra and radiation damage parameters for the Russsian BOR-60 and SM-2 reactors.

46. Shim, J.-H., et al., Atomistic modeling of the interaction between self-interstitial dislocation loops and He in bcc Fe. Journal of Nuclear Materials, 2007. 367-370: p. 292-297.

47. Lucas, G. and R. Schäublin, Stability of helium bubbles in alpha-iron: A molecular dynamics study. Journal of Nuclear Materials, 2009. 386-388: p. 360362.

48. Adams, J.B. and W.G. Wolfer, Formation energies of helium-void complexes in Nickel. Journal of Nuclear Materials, 1989. 166: p. 235-242. 